REVIEWS

\title{
The barriers preventing pregnant women from accessing midwife-led antenatal care in Nigeria
}

\author{
Clementina U. Nwankwo*1, Chidum E. Ezenwaka² \\ ${ }^{1}$ Department of Nursing Science, Faculty of Health Sciences \& Technology, Nnamdi Azikiwe University, Nnewi Campus, Nigeria \\ ${ }^{2}$ Department of Para-Clinical Sciences, Faculty of Medical Sciences, The University of the West Indies, St Augustine Campus, \\ Trinidad and Tobago
}

Received: October 29, 2019

Accepted: January 13, 2020

Online Published: February 21, 2020

DOI: $10.5430 /$ jnep.v10n5p36

URL: https://doi.org/10.5430/jnep.v10n5p36

\begin{abstract}
Background: Nigeria records high maternal deaths despite available antenatal care services in the country. This review aims to synthesise literature on barriers affecting access to midwife-led antenatal care (ANC) among pregnant women in Nigeria.

Methods: This is a systematic literature review and involved searching of studies done in Nigeria and published between 2007 and 2018. MEDLINE 1946 to present, CINAHL, JBI, and Pubmed Central were electronically searched to identify studies on barriers affecting access to midwife-led ANC among pregnant women in Nigeria. Reference lists were hand-searched, Grey literature; Google scholar, and NuSearch e-dissertation were also searched. Citation chaining was also used. Twenty articles met the inclusion and exclusion criteria while only seven out of the twenty were included in the review after quality appraisal.

Results: The data synthesis of the literature review showed disproportionate absence of the use of midwife-led ANC in this population. Several barriers were identified in the various works reviewed. The identified core barriers are economic relating to issues like high costs of services, poverty, unemployment, financial barriers; personal barrier relates to lack of autonomy, greater convenience of using other services and personal preference, low maternal educational levels, lack of information on healthcare services/ignorance about required health services, maternal age, marital status, parity/number of children; environmental relates to distance to health facility, location of residence/rural dwellers, geographical location, lack of transportation, and farness of ANC service provider; and cultural barriers relates to husband's permission, culture of patriarchy, traditional beliefs, cultural sensitivity, cultural perception of the role of TBAs, ethnicity.

Conclusions: This review highlights the need to thoroughly explore and address context-based barriers affecting access to midwife-led ANC in order to make safe motherhood a reality in Nigeria. The principal solution to these identified barriers is antenatal education by midwives which will help to empower women thereby increasing their access to midwife-led ANC. Recommendations: Government/Policy makers should allocate sufficient fund to maternal healthcare and health promotion. They should improve ANC use by making it available, accessible and affordable to all women in Nigeria irrespective of their area of residence. Also incorporate in the educational curriculum of the approved schools of midwifery in Nigeria the suggested midwifery strategies such as named midwife, team midwife, caseload midwifery.Midwives should avail themselves of update courses in midwifery; play the necessary roles of a midwife especially being women-centered; develop interest in conducting midwifery research and using the best available evidence in their practice.
\end{abstract}

Key Words: Midwife-led ANC, Barriers, Access, Pregnant women, Nigeria

${ }^{*}$ Correspondence: Clementina U. Nwankwo; Email: amakanwankwo114@yahoo.com; Address: Department of Nursing Science, Faculty of Health Sciences \& Technology, Nnamdi Azikiwe University, Nnewi Campus, Nigeria. 


\section{BACKGROUND}

Pregnancy is associated with a large number of normal physiological changes, affecting every system of the pregnant body which may also be stressful for some women. ${ }^{[1]}$ Avoiding unnecessary interventions in normal pregnancy as well as recognising risk factors requiring active management is imperative. Risks are even greater for women with pre-pregnancy health conditions. $^{[1]}$ A core aim of midwifery care is to assess risk, and plan for any potential complication by preventing, monitoring, identifying and treating them as earlier as possible and appropriately. ${ }^{[1,2]}$ Moreover, some researchers assert that accessing adequate antenatal care (ANC) during pregnancy is necessary for maternal wellbeing and foetal growth, and it connects the woman and her family to a health facility, raises the possibility of accessing a skilled healthcare provider at birth most importantly a midwife. ${ }^{[3]}$ Indeed absent or poor access to midwife-led care during this time jeopardises a crucial link in the continuum of care, and could result in maternal death. ${ }^{[3]}$ Once more, ANC aims to reduce maternal deaths, particularly in places with high records of maternal death ${ }^{[2]}$ such as Nigeria. Studies in Nigeria document a link between maternal death and non-access of ANC. ${ }^{[4,5]}$ Nigerian women who had no ANC during pregnancy have greater risk of maternal death. ${ }^{[6,7]}$

Maternal death is indeed a crucial public health concern in many developing countries, Nigeria inclusive, and there is a possibility of identifying the causes of maternal death, such as postpartum haemorrhage, anaemia, and toxiamia/eclampsia. ${ }^{[2]}$ Evidence shows that community enlightenment concerning the importance of using midwifeled ANC and health facility delivery services, with improved quality of skilled maternity care will significantly curb these preventable causes of maternal mortality. ${ }^{[8]}$ Still, two decades following the initiation of safe motherhood, maternal deaths remain very high in Nigeria, and such deaths are likely to continue increasing unless appropriate steps are taken to improve uptake of ANC, thereby reducing unbooked emergencies. ${ }^{[9]}$ Thus, access to midwife-led ANC is the key step to reduce maternal mortality.

Midwife-led ANC can be helpful in saving the life of women. Evidence show that the lack of access to midwife-led ANC is often linked with a greater risk of complications and maternal mortality. ${ }^{[10,11]}$ Antenatal care offers chance of caring for pregnant women, preventing and treating diseases, and the perception of women about this service is imperative. ${ }^{[12]}$ From literature, ANC offers recognisable opportunities to improve the health of women who access it. Unfortunately, only few pregnant women worldwide receive a minimum of four antenatal visits as recommended by the WHO. ${ }^{[13]}$

Published by Sciedu Press
Many countries including Nigeria have implemented programmes to provide ANC during pregnancy and ensure delivery by a skilled health attendant, particularly midwives. ${ }^{[14]}$ Initiation of midwife service scheme (MSS) by the Nigerian government is a good example. ${ }^{[14]}$ Worldwide, the percentage of pregnant women who receive ANC at least once was $83 \%$ for 2007 and 2014 period. ${ }^{[15]}$ Only $64 \%$ of the women received the recommended minimum of four ANC visits or more, indicating the need of fostering expansions in ANC coverage. ${ }^{[15]}$ Access to midwife-led ANC can offer appreciable benefits of improving maternal health. Improving ANC is essential and has been pertinent toward attaining the Millennium Development Goals (MDGs), now sustainable development goals (SDGs) ${ }^{[16]}$ Antenatal care is a key element of maternal health and consists of a varied range of activities with recognisable potential benefits for favourable pregnancy outcomes. ${ }^{[17,18]}$ A study reports that women who access care provided by midwives have great levels of satisfaction, and midwife-led services result in lower costs due to fewer needless, advanced technological interventions. ${ }^{[19]}$ This review aims to promote maternal health and reduce maternal death in Nigeria by exploring the barriers affecting pregnant women accessing midwife-led ANC in Nigeria. By broadening the scholarly discussion of such an important topic, this review will create a deeper understanding of the existence and nature of barriers to the use of ANC, thus providing valuable assistance to researchers and practitioners. It will help inform midwives in Nigeria and in the other developing countries especially where maternal mortality rate is still high. It will also help to educate policy makers of the benefits of midwife-led ANC especially in promoting maternal health, as well as the existing barriers to accessing midwife-led ANC. If the identified barriers are addressed by government, midwives and other healthcare providers, it may improve maternal health and reduce maternal mortality which has high prevalence rate in the country.

The following objectives guide the realization of the above aim.

I. Critically analyse the women's reasons for not accessing midwife-led ANC during pregnancy in Nigeria.

II. Ascertain the women's awareness of the available midwife-led ANC in Nigeria.

III. Critically evaluate the existing research in developing evidence for clinical practice at antenatal clinics in Nigeria. IV. Critically evaluate the use of systematic review in developing evidence for midwifery practice.

$\mathrm{V}$. State the implications of the findings of this review for maternal healthcare in Nigeria, and offer useful recommendations as appropriate. 


\subsection{Type of review for this study}

Systematic review (SR) of the literature systematically search for, appraise and synthesis various research evidence, following SR guidelines. ${ }^{[20,21]}$ A SR of literature can raise the standards of clinical research, offers evidence of its impact on experimental results, improves current practices, internal validity and reporting quality, and also reduces publication and reporting bias. ${ }^{[22]}$ A SR of literature motivates researchers to undertake studies very critically and maintain consistency in prioritising empirical evidence over predetermined knowledge. ${ }^{[23]}$ A SR of literature is also a progressively significant publication type for making informed health policy and patient care decisions. ${ }^{[24,25]}$ Both qualitative and quantitative studies on barriers affecting access to midwife-led ANC in Nigeria were sort.

\subsection{Search strategy}

The search strategy for this review involves the characteristics of the study, framing the review question, and stating the inclusion and exclusion criteria for the review.
The study characteristics include PICo that is, population, phenomenon of interest and context and is used for qualitative systematic literature-based review question, study design, participants, setting, phenomenon of interest; and report characteristics for instance years considered, language. ${ }^{[26]}$

\subsection{Research question}

What are the barriers affecting pregnant women accessing midwife-led antenatal care in Nigeria?

Table 1. Framing the question

\begin{tabular}{|c|c|c|}
\hline$\overline{\mathbf{P}}$ & Population & Pregnant women \\
\hline I & $\begin{array}{l}\text { Phenomenon of } \\
\text { Interest }\end{array}$ & $\begin{array}{l}\text { Barriers to accessing midwife-led } \\
\text { ante natal care }\end{array}$ \\
\hline Co & Context & $\begin{array}{l}\text { Community- or hospital- based } \\
\text { care in Nigeria }\end{array}$ \\
\hline
\end{tabular}

The reviewers considered the following inclusion and exclusion criteria (see Table 2) which ensured a selection of relevant papers that address the review question which were then appraised for quality before inclusion in the review.

Table 2. Inclusion and exclusion criteria

\begin{tabular}{lll}
\hline ITEMS & INCLUSION CRITERIA & EXCLUSION CRITERIA \\
\hline Design & Research papers published in peer-reviewed journals. & Commentaries or editorial papers \\
Participants & Nigeria pregnant women & Pregnant women from other countries \\
Phenomenon of & Barriers to accessing midwife-led ANC in community or & Obstetricians, TBAs or maternity nurses \\
Interest & hospital settings. & Research papers from other countries \\
Setting & Research papers from Nigeria & Papers published before 2007 and after 2018 \\
Year & Research papers published between 2007 and 2018 only & Articles reported in non-English languages \\
Language & Articles reported in English language only & \\
\hline
\end{tabular}

\section{METHOD}

Various electronic databases were searched for studies done in Nigeria and published between 2007 and 2018. The studies were searched using the electronic databases: MEDLINE 1946 to present, PubMed Central, CINAHL and JBI.

\subsection{Search terms}

The following search terms were used: midwife-led antenatal care, barriers, pregnant women, community- or hospitalbased care, and Nigeria along with their synonyms/related terms were used in the search. Boolean operators (OR, AND), truncation, phrase searching and wild cards were used to improve the effectiveness of the searching. ${ }^{[27]}$ Then all relevant papers were combined to narrow the field. More so, reference lists were hand-searched. Grey literature; Google scholar, and $\mathrm{NuSearch}$ e-dissertation were also searched. Citation chaining was used to reach other relevant papers cited in the original papers. $^{[28]}$

\subsection{Selection of studies}

All searched articles were assembled into reference management software (END NOTE uRL) and any duplicated article was removed. The reviewers assessed all the potential studies identified for inclusion against the stated inclusion criteria for this review. Those studies that met the inclusion criteria were then fully retrieved and the details assessed by the reviewers against the inclusion criteria. All the studies that failed to meet the inclusion criteria were excluded from the study. A Preferred Reporting Items for SRs is a study flow diagram. ${ }^{[29]}$ This format was used to map the systematic review process for this study.

\subsection{Data extraction and management}

The data extraction form was used to put down the following details: authors and date, title, methodology, location, aims, study population and sample size, method and data analysis, and findings: barriers to midwife-led ANC. For all the eligible studies, their abstract and full text, the reviewers extracted the data using the already designed form. Then the 
most frequent barriers to accessing midwife-led ANC were used to form categories.

\subsection{Assessment of risk of bias in included studies}

The reviewers assessed risk of bias for each study using the criteria outlined in the Cochrane Handbook for Systematic Reviews of Interventions. ${ }^{[30]}$ However, all the papers that met inclusion criteria with low risk of bias were then subjected to a quality appraisal process.

\subsection{Quality appraisal}

In assessing the quality of the body of evidence, all identified studies underwent a quality appraisal by the reviewers using appropriate tool, Critical Appraisal Skill Programme (CASP Tool) for qualitative study and Center for Evidence-Based Management (CEBM) for quantitative study (cross-sectional study/survey). Studies that gained reviewers' agreement were included and any discrepancy that arose was resolved by discussion.

\subsection{Data synthesis}

New JBI SUMARI was used for data synthesis (Narrativesynthesis). The data synthesis in this review involves the following: using data extraction method to identify and extract data from included studies, consider the most frequent and most common data, and categorise them. ${ }^{[31]}$ that is: precisely, grouping/aggregating the data into categories on the basis of similarity in meaning, and finally, synthesising the categories to create "synthesised data". ${ }^{[32]}$

\subsection{Ethical considerations}

Systematic Review is a secondary research studies and do require the reviewers to be ethical. Honesty in communicating information about the review and not falsifying or misrepresenting data; reliability by being meticulous in disseminating the results fairly and fully without bias; objectivity by designing the study to minimise bias and also actively avoid bias in data analysis and interpretation as well; transparency in dealing with facts and data; and fairness in relation to references and proper acknowledgement of sources used. ${ }^{[33,34]}$ Besides, the reviewers followed a systematic approach.

\section{RESULTS}

\subsection{Results of the search}

Four databases: CINAHL, JBI, PubMed Central, and 'MEDLINE Plus 1946 to present' were properly searched. A total of 836 studies ( 829 from electronic searches, 4 from Google scholar and 3 from hand searching the reference list) were identified and exported to reference management software (END NOTE). A total of 452 duplicates were removed, leaving 384 studies to be assessed. The titles and abstracts of the remaining studies were closely reviewed against the inclusion and exclusion criteria and 364 studies were excluded remaining 20 papers (see Figure 1).

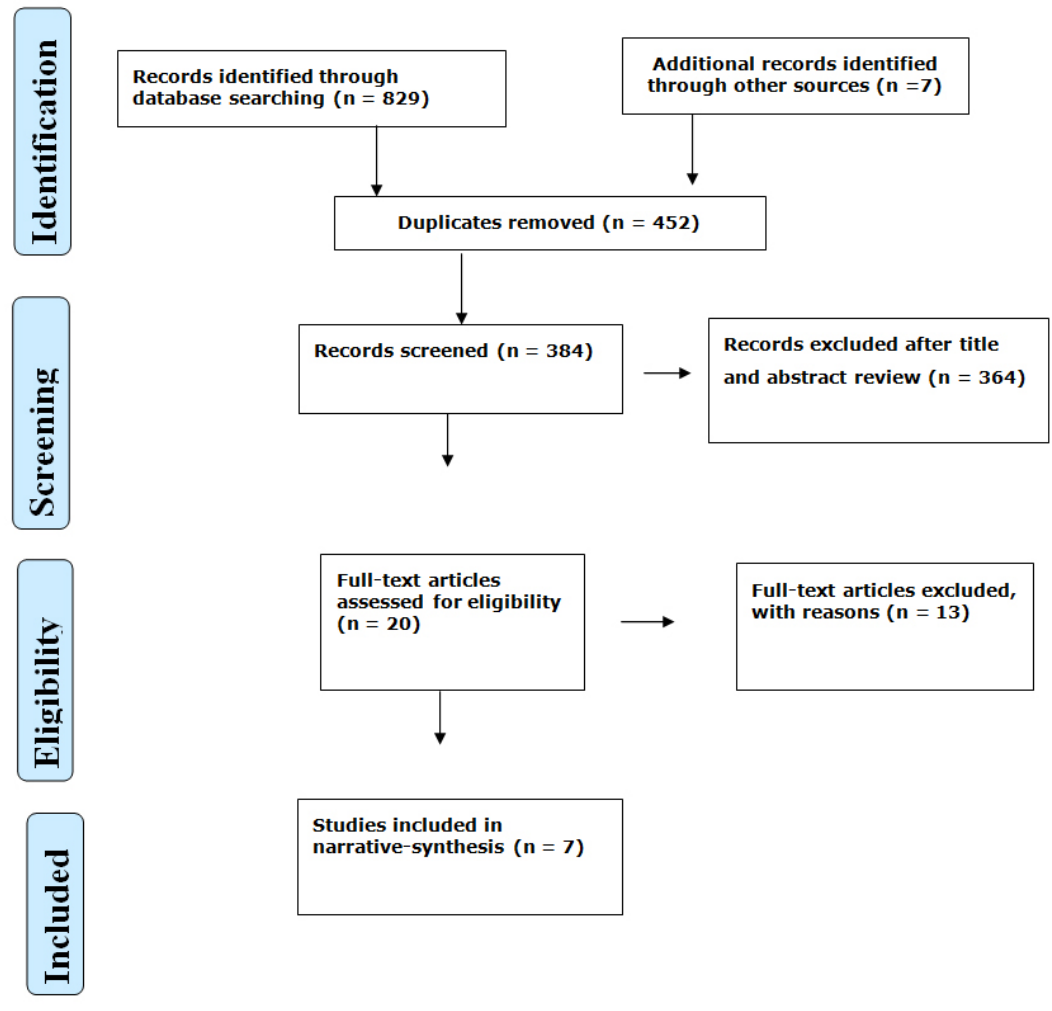

Figure 1. Prisma 2009 Flow Diagram ${ }^{[35]}$ 


\subsection{Result of quality appraisal}

The Critical Appraisal Skills Programme (CASP) and the Center for Evidence-Based Management (CEBM) Tools were used to independently appraise the quality of each paper for inclusion in the review. CASP is used to appraise the qualitative research papers. ${ }^{[36]}$ CEBM helps to select good quality cross-sectional papers. ${ }^{[37]}$ The reviewers decided that studies needed to rate 'yes' for more than half of the total questions in CASP Tool and CEBM to be considered of good quality. Out of the 20 studies appraised 13 did not meet the quality appraisal threshold of above 50\% 'Yes', while seven (7) papers met the threshold and were included in the review. CASP has a total of 10 questions while CEBM has a total of 12 questions and all the questions have equal points. The points attained by each paper after appraisal is converted to percentage. This helps to ensure that both qualitative and quantitative papers are rated equally without bias. Decision rule: any paper that has a score from $60 \%$ to $100 \%$ is considered for inclusion in the review and any paper that has a score below $60 \%$ is excluded. This is because scores between $59 \%$ and below are deemed 'Fail'. ${ }^{[38]}$ The seven papers that are good for inclusion have clear details about their methodology, method of data collection, and their findings included the quotes from the women. The thirteen papers that were deemed poor failed to give clear details about their design, methodology, method of data collection, and did not include qualitative text from the participants in their findings. The characteristics of studies included in the review are shown in Table 3 (see the Appendix).

\subsection{Data extraction and meta-synthesis}

The Data Extraction Form designed by the reviewers based on CASP tool was used to extract findings from the included seven studies of five qualitative and two quantitative papers. A total of 44 findings were extracted which are presented in a Data Extraction Matrix. The findings were classified into eight categories based on their similarities in meaning (wording and concept), and each category represents one analytical theme for clarity. Some of the participants' voices (direct quotes) as obtained from the papers were included in this review to authenticate the findings. The categorised findings are as follows:

1) Personal barriers: lack of autonomy, greater convenience of using other services and personal preference, low maternal educational levels, lack of information on healthcare services/ignorance about required health services, maternal age, marital status, parity/number of children, relative autonomy of women or empowerment, and woman's assessment of quality of care.

2) Economic barriers: high costs of services, poverty, unemployment, financial barriers, economic constraint, affordability of services, poor wealth quintile.

3) Cultural barriers: husband's permission, culture of patriarchy, traditional beliefs, belief that it may not be necessary or customary, cultural sensitivity, cultural perception of the role of TBAs, and ethnicity.

4) Health facility management barriers: lack of essential drugs and equipment, long waiting hours/delay, few working hours, absence of staff/availability of care particularly at night, healthcare workers strike, and time/health facility being closed at night.

5) Social barriers: negative attitude of staff, and social support. 6) Environmental barriers: distance to health facility, location of residence/rural dwellers, geographical location, lack of transportation, and farness of ANC service provider. 7) Psychological barriers: low self-esteem, abuse, fears of surgery, fears of other hospital interventions, inability to go alone, trust in service quality, and previous negative experiences with health facilities.

8) Physiological barriers include: onset of labour at night.

The eight categories of synthesised findings were made distinctive by their frequency of occurrence as presented in the summary of data extraction matrix (see Table 4). Thus, emerged four core categories or themes and can be used as a basis for EBP and evidence-based recommendations relating to barriers affecting access to midwife-led ANC among pregnant women.

Table 4. Summary of the Data Extraction Matrix (Total number of findings = 44)

\begin{tabular}{llll}
\hline Categorised barriers & Number of findings & Frequency of occurrence & Ranking/Remarks \\
\hline Personal & 9 & 14 & $2^{\text {nd }}($ Core barriers $)$ \\
Economic & 7 & 16 & $1^{\text {st }}($ Core barriers $)$ \\
Psychological & 7 & 7 & $5^{\text {th }}$ \\
Cultural & 7 & 10 & $4^{\text {th }}($ Core barriers $)$ \\
Social & 2 & 4 & $7^{\text {th }}$ \\
Environmental & 5 & 12 & $3^{\text {rd }}($ Core barriers $)$ \\
Health facility management & 6 & 7 & $5^{\text {th }}$ \\
Physiological & 1 & 1 & $8^{\text {th }}$ \\
\hline
\end{tabular}


Theme one: Economic barriers constitute the key barriers to accessing midwife-led ANC in Nigeria. Some participants' quotes include: "our problem is poverty; our daily struggles relate to feeding our families. God save you if you the wife now needs surgery which can costs thousands of Naira... the simple truth is government has to help us". [39] "I was sick during pregnancy and I went to the State Hospital, they wrote me a bill of over four thousand Naira when they hadn't even given me a single medication..... [New Mother]. ${ }^{[40]}$ "When you come for antenatal they ask you to buy drug, or to do urine test, you will pay for all those things. In the maternity home, you don't really spend much, maybe that scare women away from the hospital." ${ }^{[41]}$ Women reported that sometimes they may not get the attention of the healthcare provider if unable to "pay before service". ${ }^{[41]}$ 'Sincerely, the advantage of giving birth at home is that it saves money. It doesn't involve mandatory buying of tissue, Dettol or other things'. ${ }^{[40]}$

Theme two: Personal barriers are among the key barriers to accessing midwife-led ANC in Nigeria. Some participants made the following quotes:"Some are advised by their mother-in-law ... when women are not educated they yield to whatever advice they are given". ${ }^{[41]}$ "I cannot go to the health facility for care during pregnancy because am not sick".[42 P.969] "Some people believe is only God that can help to deliver and so prefer to stay back at home for delivery, besides it is very comfortable and at no cost" "[42] "Yes, attending for ANC is important but you only go there to deliver if you become weak or cannot deliver on your own without assistance ... One goes there only if a problem develops or when the labour takes much time"[39] "Some women prefer traditional home because if there is difficulty during delivery, spiritual intervention can be obtained" [42] "Allah (God) has now brought succor in ANC. Labor is now short, quick and uncomplicated... [39 P.589] And... If " you go to the hospital for delivery, people will think problems have emerged and you need assistance". Others were outrightly spiritual... "We don't have problems now. Allah is helping us"[39]

Theme three: Environmental barriers. Quotes by some participants are: 'when labour started, we couldn't get a machine [motorcycle] to bring us here [...]. And again, the labour has intensified, and as a result, I couldn't walk. And so I have no option again than to give birth at home. So the people around me took care of me because our house is far from here [the clinic]. ${ }^{[43]}$

Theme four: Cultural barriers. Some quotes from the women include:"If there is no approval from husband, we would not go to seek care"[42] "A lot of women rely on their husbands and/or mothers-in-law for decision-making ...” “... many women are housewives. "There is one in my area ... she is pregnant ... in fact she is supposed to be here today, the man doesn't give her money for food, does not even take care of her and all that ... You know it is frustrating". ${ }^{41]}$

\section{Discussion}

\section{Theme one: Economic barriers}

The review shows that women do not access midwife-led ANC mainly due to lack of economic power. Evidence show that poor economic status ${ }^{[44]}$ and financial constraints ${ }^{[45]}$ are barriers to accessing midwife-led ANC. Pregnant women need money to care for their needs and lack of money is a barrier to accessing ANC. ${ }^{[46,47]}$ There is an inextricable link between poverty and poor health, and women enduring poverty are also usually less educated. ${ }^{[48]}$ Such women have low knowledge about activities to promote health and when to access health care; for instance, poor women access midwife-led ANC less frequently and suffer poorer birthing outcomes than wealthy women. ${ }^{[48,49]}$ Cost is a barrier to accessing midwife-led ANC, and a reason to visit a TBA because of flexible payment. ${ }^{[50]}$ Indeed, wealth significantly influences proper uptake of midwife-led ANC. ${ }^{[51]}$ Evidence shows that socioeconomic status of women influences ANC service use when women perceive the benefits of the service to outweigh the cost. ${ }^{[52]}$ Contrarily, women especially rural dwellers access TBA-model of care because of its availability, accessibility, and affordability. ${ }^{[53]}$ Arguably, some researchers assert that policy-makers should endeavour to encourage pregnant women to have regular prenatal care services and also assist in lowering out-of-pocket fees for low-income women. ${ }^{[54]}$ This helps to make midwife-led ANC readily available, accessible and affordable too.

\section{Theme two: Personal barriers}

In general an individual controls his/her health status thus the Theory of Reasoned Action and Theory of Planned Behaviour explain and predict numerous health behaviours and intentions such as health services utilisation. ${ }^{[55]}$ Precisely, many women who have good health behaviour and intention to access ANC are faced with some personal challenges as revealed in this review such as low maternal education/illiteracy. Evidence show that low maternal education is a barrier ${ }^{[44,56]}$ and education improves women's knowledge and increases their earnings. ${ }^{[57]}$ Evidence shows that illiteracy is a barrier to accessing ANC. ${ }^{[58]}$ Some women have lack of awareness on delivery care, and cost. ${ }^{[59,60]}$ and also lack of knowledge about available services. ${ }^{[45]}$ Supportably, evidence report that low level of education is associated with non-attendance to childbirth and parenthood education classes during pregnancy. ${ }^{[61]}$ More so, an educated woman is capable of making decision on her own about antenatal 
visit. ${ }^{[62]}$ and she is more likely to attend ANC. ${ }^{[63,64]}$ Evidence shows that maternal education is linked with the use of ANC in Ethiopia and women with higher education attainment access ANC better than their counterparts. ${ }^{[65]}$ Mother's education is the most important factor that links with the use of ANC and skilled attendance at delivery in India. ${ }^{[66]}$

\section{Theme three: Environmental barriers}

This theme focuses on barriers that affect women reaching the health facility to access midwife-led ANC. A study reports such barriers to include transport among others. ${ }^{[50]}$ Previous researchers report that long distances and lack of transport constitute constraints to accessing ANC by women in Zambia. ${ }^{[67]}$ Distance to hospital and lack of transportation. ${ }^{[59,60]}$ and accessibility of care constitutes a barrier to midwife-led ANC use. ${ }^{[68]}$ Evidence shows that women access ANC service more when the service is within reasonable distance of the women's place of residence. ${ }^{[52]}$ Women who reside in urban areas do access health facility under skilled provider such as a midwife while those in the rural areas indulge in home care under unskilled personnel. ${ }^{[69]}$ In addition, some researchers assert that barriers to accessing midwifeled ANC include walking long distances to access health facilities among others. ${ }^{[70]}$

\section{Theme four: Cultural barriers}

Culture can be defined as "the way of life of a group: the learnt patterns of behaviour that are socially constructed and transmitted" such as a shared communication system in language, gestures and expressions, values and ideas. ${ }^{[71]}$ The culture of the society poses a challenge to women access to midwife-led ANC. Cultural barriers include cultural beliefs and practices like traditional birthing practices. ${ }^{[72-74]}$ and husband's preference. ${ }^{[50]}$ Culture of patriarchy poses a great problem in Nigeria. The Nigerian society adopts patriarchal culture which allows men to dominate women ${ }^{[75]}$ and the father as patriarch is being highly respected. ${ }^{[76]}$ Women access to midwife-led ANC is greatly affected by a husband's approval or permission, ${ }^{[77,78]}$ husbands' social support ${ }^{[79]}$ and objection from family. ${ }^{[45]}$ A study reports that lack of capacity building and sensitisation is a key element of economic challenge among women, therefore a review of strategies used for policy implementation in some of the African societies is necessary to empower women. ${ }^{\left[{ }^{80]}\right.}$ Evidence indicates the need to curb all forms of inhuman discrimination and gender inequality. ${ }^{[75]}$ This will give the women opportunity to decide on their own health issues. In relation to cultural perception of the role of TBAs, evidence shows that women perceive that TBAs play a role in cultural competence, consolation, empathy and psychosocial support. ${ }^{[81]}$ Thus, they patronise TBAs.
In overall, the findings of this review are quite different from what happens in developed countries like United Kingdom. For instance a study conducted in UK reports that women's initial access is influenced by late pregnancy recognition and subsequent denial or acceptance while their ongoing access depends on the perceived gains and losses from ANC. ${ }^{[82]}$ Moreover, personal barriers such as time, money and social support, alongside the perceived quality of care, the trustworthiness and cultural sensitivity of staff, and feelings of mutual respect are also crucial. ${ }^{[82]}$ Contrarily, a study documents that reasons for late initiation of ANC in England and Wales include maternal age at booking, smoking status, ethnicity, type of hospital at booking, the planned pattern of ANC and the planned place of delivery. ${ }^{[83]}$

\subsection{Implications for improving midwifery practice and informing health policy}

The findings of this review present vital implications for improving midwifery practice, especially in Nigeria as women are disempowered to access midwife-led ANC, but also for midwifery practice worldwide. Women empowerment is necessary in accessing midwife-led ANC and empowering women is a role of a midwife. Empowerment is a dynamic and enabling process of acquiring freedom and power, particularly for socially marginalised persons and groups. ${ }^{[84,85]}$ and empowerment also enables people to gain control over their lives through raising awareness, taking action and working towards exercising greater control. ${ }^{[85]}$ The identified barriers like personal and cultural can be addressed by the midwife simply by empowering women. The midwife plays this role by proper antenatal education to women in order to enrich the women's knowledge. Knowledge is said to be power. Midwife should give the women health education about prevention and earlier detection of the causes of maternal mortality in Nigeria. Midwife can meet women in their homes, assess their homes and make friends with the women and family. ${ }^{[86,87]}$ Thus, promoting the women's trust on the midwife and midwife-led ANC, and discourages women from accessing TBA-led care. More so, midwives can involve TBAs in ANC education, this helps to get TBAs on board and involved in maternal services. ${ }^{[88,89]}$ Women can be personally invited to meet midwives and this helps the midwife have enough time for personalising the woman's encounter in $\mathrm{ANC} ;{ }^{[90]}$ Midwife can engage in one-to-one ANC education with women and this offers a continuous and personal relationship between each woman and her midwife; ${ }^{[91]}$ there could be named midwives who provide their contact details to women, and team midwives can also be established who see the women very often than named midwives; ${ }^{[92]}$ case holding, ${ }^{[93]}$ better birth, ${ }^{[94]}$ and changing childbirth; ${ }^{[95]}$ Midwife can organise ANC education at the community 
and women to come in groups and this allows women build friendships and support networks; ${ }^{[96]}$ guideline- and networkbased strategy ${ }^{[97]}$ are good approaches too. All these strategies which are readily available in developed countries can go a long way in empowering women if practiced in Nigeria. Thus, enhancing adequate access to midwife-led ANC, improve maternal health, and decrease maternal death which is recorded high in Nigeria.

The health policy makers and government should partly empower women to access midwife-led ANC by addressing mainly the environmental and economic barriers. For instance, the MSS in Nigeria is a good policy, although it needs to be intensified. Gender equality policy should be reinforced, and women be allowed to assume certain essential roles and economic power. Sustainable Development Goals on poverty eradication, improving girl-child education, and improving maternal health should be reinforced. There is need for health facility to be made accessible and available to women irrespective of their areas of residence especially women living in rural areas. More health facilities should be established in the rural areas where majority of the women are in dire need of midwife-led ANC.

In overall, these proffered solutions could be extended in the future to ascertain whether Nigerian women can access midwife-led ANC.

\subsection{Summary}

The identified barriers were quite different from barriers in developed countries like United Kingdom. Implications for improving midwifery practice focuses on antenatal health education by midwives to empower women. Other possible solutions such as use of named midwives, caseload midwifery were stated. This review also has implications for formulating health policy on intensfying MSS in Nigeria, reinforcing gender equality among others. Overall, there is need for women empowerment in Nigeria to enhance access to midwife-led ANC.

\section{Conclusion}

This review sought to proffer solution to the barriers affecting access to midwife-led ANC among pregnant women in Nigeria. Based on the objectives of the review, the following conclusions can be adduced:

1) Nigerian women are aware of the availability of midwifeled ANC.

2) Some existing research studies develop evidence regarding clinical practice at antenatal clinics in Nigeria (the 7 included papers) though these are primary studies, and there is need for SRs.

3) It is imperative to use SR in the development of evidence for midwifery practice in Nigeria.

4) This review provides implications for improving midwifery practice and informing policy in Nigeria, and also offers useful recommendations.

\section{Recommendations}

Government/Policy makers should allocate sufficient fund to maternal healthcare and health promotion. They should improve ANC use by making it available, accessible and affordable to all women in Nigeria irrespective of their area of residence. Also incorporate in the educational curriculum of the approved schools of midwifery in Nigeria the suggested midwifery strategies such as named midwife, team midwife, caseload midwifery. Midwives should avail themselves of update courses in midwifery; play the necessary roles of a midwife especially being women-centered; develop interest in conducting midwifery research and using the best available evidence in their practice.

\section{CONFlicts OF INTEREST Disclosure}

The authors declare that there is no conflict of interest.

\section{REFERENCES}

[1] Meng CT, Singh K. Routine antenatal care. Integrated Approach to Obstetrics and Gynaecology. 2016; 305. https ://doi .org/10.1 142/9789813108561_0022

[2] Carroli G, Rooney C, Villar J. How effective is antenatal care in preventing maternal mortality and serious morbidity? An overview of the evidence. Paediatric and Perinatal Epidemiology. 2001; 15(s1): 1-42. PMid:11243499 https://doi.org/10.1046/j.1365-301 $6.2001 .0150 \mathrm{~s} 1001 . \mathrm{x}$

[3] Lincetto O, Mothebesoane-Anoh S, Gomez P, et al. Antenatal care. Opportunities for Africa's newborns: Practical data, policy and programmatic support for newborn care in Africa. 2006; 55-62.
[4] Andersson N, Omer K, Caldwell D, et al. Male responsibility and maternal morbidity: a cross-sectional study in two Nigerian States. BMC Health Services Research. 2011; 11(2): S7. PMid:22375828 https://doi.org/10.1186/1472-6963-11-S2-S7

[5] Mairiga AG, Saleh W. Maternal mortality at the state specialist hospital Bauchi, Northern Nigeria. East African Medical Journal. 2009; 86(1). PMid:19530545 https://doi.org/10.4314/eamj .v86i1.46924

[6] Ezugwu EC, Agu PU, Nwoke MO et al. Reducing maternal deaths in a low resource setting in Nigeria. Nigerian Journal of Clinical Practice. 2014; 17(1): 62-6. PMid:24326810 https://doi.org/ 10.4103/1119-3077.122842

[7] Doctor HV, Findley SE, Cometto G, et al. Awareness of critical dan- 
ger signs of pregnancy and delivery, preparations for delivery, and utilization of skilled birth attendants in Nigeria. Journal of Health Care for the Poor \& Underserved. 2013; 24(1): 152-70. PMid:23377725 https://doi.org/10.1353/hpu. 2013.0032

[8] Kullima AA, Kawuwa MB, Audu BM, et al. Trends in maternal mortality in a tertiary institution in Northern Nigeria. Annals of African Medicine. 2009; 8(4): 221-4. PMid:20139543 https: //doi.org/10.4103/1596-3519.59575

[9] Igberase G, Ebeigbe PN. Maternal mortality in a rural referral hospital in the Niger Delta, Nigeria. Journal of Obstetrics \& Gynaecology. 2007; 27(3): 275-278. PMid:17464810 https://doi.org/10.1 080/01443610701213687

[10] Osazua H. Benefits of antenatal care. 2016. Wellspring hospital, Abuja-FCT.

[11] Bauserman M, Lokangaka A, Thorsten V, et al. Risk factors for maternal death and trends in maternal mortality in low- and middleincome countries: a prospective longitudinal cohort analysis. Reproductive Health. 2015; 12 Suppl 2: S5. PMid:26062992 https: //doi.org/10.1186/1742-4755-12-S2-S5

[12] Ekott MI, Ovwigho U, Ehigiegba A et al. Perception of pregnant women about antenatal care in a cottage hospital in Port Harcourt, Nigeria. Journal of Community Health. 2013; 38(2): 360-365. PMid:23054423 https://doi.org/10.1007/s10900-012-962 5-1

[13] WHO. World health statistics 2010. 2010; World Health Organization.

[14] Oyetunde MO. Quality issues in midwifery: A critical analysis of midwifery in Nigeria within the context of the International Confederation of Midwives (ICM) global standards. International Journal of Nursing and Midwifery. 2014; 6(3): 40-48. https: //doi.org/10.5897/I JNM2013-0119

[15] WHO. World health statistics 2015. 2015; World Health Organization.

[16] Banchani E, Tenkorang EY. Occupational types and antenatal care attendance among women in Ghana. Health Care for Women International. 2014; 35(7-9): 1040-1064. PMid:24850372 https: //doi.org/10.1080/07399332.2014.919581

[17] Aliyu AA, Dahiru T. Predictors of delayed Antenatal Care (ANC) visits in Nigeria: secondary analysis of 2013 Nigeria Demographic and Health Survey (NDHS). The Pan African Medical Journal. 2017; 26. https://doi.org/10.11604/pamj .2017.26.124.9861

[18] Nuraini E, Parker E. Improving knowledge of antenatal care (ANC) among pregnant women: a field trial in central Java, Indonesia. Asia Pacific Journal of Public Health. 2005; 17(1): 3-8. PMid:16044824 https://doi.org/10.1177/101053950501700102

[19] Rosy MM. Evidence based Practice in Midwifery. International Journal of Nursing Education and Research. 2016; 4(3): 376-382. https://doi.org/10.5958/2454-2660.2016.00067.3

[20] Avellar SA, Thomas J, Kleinman R, et al. External validity: The next step for systematic reviews?. Evaluation Review. 2017; 41(4): $283-$ 325. PMid:27580622 https://doi.org/10.1177/0193841X16 665199

[21] Grant MJ, Booth A. A typology of reviews: an analysis of 14 review types and associated methodologies. Health Information \& Libraries Journal. 2009; 26(2): 91-108. PMid:19490148 https: //doi.org/10.1111/j.1471-1842.2009.00848.x

[22] Percie du Sert N. The role of systematic reviews in improving the internal validity and reporting quality of animal research. National Centre for the Replacement, Refinement \& Reduction of Animals in Research. 2017.

[23] Mallett R, Hagen-Zanker J, Slater R, et al. The benefits and challenges of using systematic reviews in international development re- search. Journal of Development Effectiveness. 2012; 4(3): 445-455. https://doi.org/10.1080/19439342.2012.711342

[24] University of California San Francisco, UCSF Library. More about pros and cons of doing a systematic review - Systematic. 2017; Available from: https://guides.ucsf .edu \T1\guilsinglri ghtUCSFGuides $\backslash \mathrm{T} 1 \backslash$ guilsinglrightLibrary $\backslash \mathrm{T} 1 \backslash$ guilsing lrightSystematicReview

[25] Murad MH, Asi N, Alsawas M et al. New evidence pyramid. BMJ Evidence-Based Medicine. 2016.

[26] Moher D, Shamseer L, Clarke M, et al. Preferred reporting items for systematic review and meta-analysis protocols (PRISMA-P) 2015 statement. BioMed Central. 2015; 4: 1. PMid:25554246 https://doi.org/10.1186/2046-4053-4-1

[27] Eastman CM, Jansen BJ. Coverage, relevance, and ranking: The impact of query operators on Web search engine results. ACM Transactions on Information Systems (TOIS). 2003; 21(4): 383-411. https://doi.org/10.1145/944012.944015

[28] Harrell Health Sciences Library, Research and Learning Commons. How do I do a systematic review? PennState College of Medicine. 2018; Available from: http://harrell.library.psu.edu/c. php?g=576583\&p=3979424

[29] Stovold E, Beecher D, Foxlee R et al. Study flow diagrams in Cochrane systematic review updates: an adapted PRISMA flow diagram. Systematic Reviews. 2014; 3(1): 54. PMid:24886533 https : //doi.org/10.1186/2046-4053-3-54

[30] Higgins JPT, Green S. Cochrane Handbook for Systematic Reviews of Interventions (2011) (Ed.) Version 5.1.0. 2011; Available from: http://handbook-5-1.cochrane.org/chapter 2/2_3_8_resources_for_a_systematic_review.htm

[31] Finlayson K, Dixon A. Qualitative meta-synthesis: A guide for the novice. Nurse Researcher. 2008; 15(2): 59. PMid:18283763 https://doi.org/10.7748/nr2008.01.15.2.59.c6330

[32] Godfrey CM, Harrison MB. Systematic Review Resource Package The Joanna Briggs Institute Method for Systematic Review Research Quick Reference Guide* Queen's Joanna Briggs Collaboration Version 4.0. 2015; Available from: https://joannabriggs.org/assets/docs/jbc/operation s/cansynthesise/CAN_SYNTHSISE_Resource-.pdf

[33] Enago academy. How Research Institutes Should Promote Ethical Behavior. 2018; Available from: https: //www. enago.com/acad emy/research-institutes-promote-ethical-behavior/

[34] ALLEA - All European Academies. The European Code of Conduct for Research Integrity Revised Edition. 2017; Berlin: ALLEA - All European Academies. Available from: https: //ec.europa.eu/research/participants/data/ref/h202 0/other/hi/h2020-ethics_code-of-conduct_en.pdf

[35] Liberati A, Altman DG, Tetzlaff J et al. The PRISMA statement for reporting systematic reviews and meta-analyses of studies that evaluate health care interventions: explanation and elaboration. PLoS Medicine. 2009; 6(7): e1000100. PMid:19621070 https: //doi.org/10.1371/journal.pmed.1000100

[36] Chenail RJ. Learning to appraise the quality of qualitative research articles: A contextualized learning object for constructing knowledge. The Qualitative Report. 2011; 16(1): 236-248.

[37] Burns PB, Rohrich RJ, Chung KC. The levels of evidence and their role in evidence-based medicine. Plastic and Reconstructive Surgery. 2011; 128(1): 305. PMid:21701348 https ://doi.org/10.1097/ PRS.0b013e318219c171

[38] Hernandez B. How To Figure a Percentage and Letter Grade. 2017; [Available online] Available from: https://www.thoughtco.co $\mathrm{m} /$ figure-percentage-and-letter-grade-1828610 
[39] Tukur I, Cheekhoon C, Tinsu T, et al. Why women are averse to facility delivery in Northwest Nigeria: a qualitative inquiry. Iranian Journal of Public Health. 2016; 45(5): 586.

[40] Akeju DO, Oladapo OT, Vidler M, et al. Determinants of health care seeking behaviour during pregnancy in Ogun State, Nigeria. Reproductive Health. 2016; 13(1): 32. PMid:27356754 https: //doi.org/10.1186/s12978-016-0139-7

[41] Ohaja M, Murphy-Lawless J. Persistent challenges and barriers encountered by women attending formal antenatal care in south east Nigeria. Africa Journal of Nursing and Midwifery. 2017; 19(2): 1-13. https://doi .org/10.25159/2520-5293/2019

[42] Abimbola JM, Makanjuola AT, Ganiyu SA, et al. Pattern of utilization of ante-natal and delivery services in a semi-urban community of North-Central Nigeria. African Health Sciences. 2016; 16(4): 962-971. PMid:28479888 https ://doi .org/10.4314/ah s.v16i4.12

[43] Exley J, Pitchforth E, Okeke E, et al. Persistent barriers to care; a qualitative study to understand women's experiences in areas served by the midwives service scheme in Nigeria. BMC Pregnancy and Childbirth. 2016; 16(1): 232. PMid:27538806 https: //doi.org/10.1186/s12884-016-1026-5

[44] Singh DR, Jha T. Exploring Factors Influencing Antenatal Care Visit Dropout at Government Health Facilities of Dhanusha District, Nepal. American Journal of Public Health Research. 2016; 4(5): 170-5.

[45] Agarwal P, Singh MM, Garg S. Maternal health-care utilization among women in an urban slum in Delhi. Indian Journal of Community Medicine. 2007; 32(3): 203. https ://doi .org/10.4103/09 70-0218.36829

[46] Mrisho M, Obrist B, Schellenberg JA, et al. The use of antenatal and postnatal care: perspectives and experiences of women and health care providers in rural southern Tanzania. BMC Pregnancy and Childbirth. 2009; 9(1): 10. PMid:19261181 https: //doi.org/10.1186/1471-2393-9-10

[47] Ganga-Limando M, Moleki M, Modiba L. Potential barriers to utilisation of maternal health services in public health facilities in rural and remote communities: a qualitative study. Life Sci J. 2014; 11(10): 973-979. https://doi .org/10.1038/nmeth. 3141

[48] Murray S. Poverty and health. Canadian Medical Association Journal. 2006; 174(7): 923-923.

[49] Peters DH, Garg A, Bloom G et al. Poverty and access to health care in developing countries. Annals of the New York Academy of Sciences. 2008; 1136(1): 161-171. PMid:17954679 https: //doi.org/10.1196/annals.1425.011

[50] Mason L, Dellicour S, Ter Kuile F et al. Barriers and facilitators to antenatal and delivery care in western Kenya: a qualitative study. BMC pregnancy and childbirth. 2015; 15(1): 26. PMid:25886593 https://doi.org/10.1186/s12884-015-0453-z

[51] Arthur E. Wealth and antenatal care use: implications for maternal health care utilisation in Ghana. Health Economics Review. 2012; 2(1): 14. PMid:22866869 https://doi.org/10.1186/2191-1 991-2-14

[52] Griffiths P, Stephenson R. Understanding users' perspectives of barriers to maternal health care use in Maharashtra, India. Journal of Biosocial Science. 2001; 33(3): 339-359. PMid:11446398 https://doi.org/10.1017/S002193200100339X

[53] Imogie AO, Agwubike EO, Aluko K. Assessing the role of traditional birth attendants (TBAs) in health care delivery in Edo State, Nigeria. African Journal of Reproductive Health. 2002; 94-100.

[54] Beeckman K, Louckx F, Putman K. Predisposing, enabling and pregnancy-related determinants of late initiation of prenatal care. Maternal and Child Health Journal. 2011; 15(7): 1067-1075.
[55] Montano DE, Kasprzyk D. Theory of reasoned action, theory of planned behavior, and the integrated behavioral model. Health Behavior: Theory, Research and Practice. 2015; 95-124.

[56] Onah HE, Ikeako LC, Iloabachie GC. Factors associated with the use of maternity services in Enugu, southeastern Nigeria. Social Science \& Medicine. 2006; 63(7): 1870-1878. PMid:16766107 https://doi.org/10.1016/j.socscimed.2006.04.019

[57] Hill K, Hoffman D, Rex TR. The value of higher education: Individual and societal benefits. William Seidman research Institute, Arizona State University, Tempe, AZ. 2005.

[58] Dhakal S, Van Teijlingen ER, Stephens J, et al. Antenatal care among women in rural nepal: a community-based study. Online Journal of Rural Nursing and Health Care. 2011; 11(2): 76-87.

[59] Dhakal S, Van Teijlingen E, Raja EA, et al. Skilled care at birth among rural women in Nepal: practice and challenges. Journal of Health, Population, and Nutrition. 2011; 29(4): 371. PMid:21957676 https://doi.org/10.3329/jhpn.v29i4.8453

[60] Ntui AN, Carson A, Turpin CA et al. Determinants of access to antenatal care and birth outcomes in Kumasi, Ghana. Journal of Epidemiology and Global Health. 2013; 3(4): 279. PMid:24206799 https://doi.org/10.1016/j.jegh.2013.09.004

[61] Fabian HM, Rådestad IJ, Waldenström U. Characteristics of Swedish women who do not attend childbirth and parenthood education classes during pregnancy. Midwifery. 2004; 20(3): 226-235.

[62] Matsumura M, Gubhaju B. Women's status household structure and the utilisation of maternal health services in Nepal. Asia-Pacific Population Journal. 2001; 16(1): 23-44. https ://doi .org/10.18356 /e8a4c9ed-en

[63] Abou-Zahr CL, Wardlaw TM, World Health Organization. Antenatal care in developing countries: promises, achievements and missed opportunities: an analysis of trends, levels and differentials, 1990-2001. 2003.

[64] Ikeako LC, Onah HE, Iloabachie GC. Influence of formal maternal education on the use of Maternity services in Enugu, Nigeria. Journal of Obstetrics and Gynaecology. 2006; 26(1): 30-34. PMid:16390706 https://doi.org/10.1080/01443610500364004

[65] Mekonnen Y, Mekonnen A. Factors influencing the use of maternal healthcare services in Ethiopia. Journal of Health, Population and Nutrition. 2003; 374-382.

[66] Jat TR, Ng N, San Sebastian M. Factors affecting the use of maternal health services in Madhya Pradesh state of India: a multilevel analysis. International Journal for Equity in Health. 2011; 10(1): 59 PMid:21492481 https://doi.org/10.1186/1476-511X-10-5 9

[67] Stekelenburg J, Kyanamina S, Mukelabai M et al. Waiting too long: low use of maternal health services in Kalabo, Zambia. Tropical Medicine \& International Health. 2004; 9(3): 390-398. PMid:14996369 https://doi .org/10.1111/j.1365-3156.20 04.01202.x

[68] Boerleider AW, Wiegers TA, Manniën J, et al. Factors affecting the use of prenatal care by non-western women in industrialized western countries: a systematic review. BMC Pregnancy and Childbirth. 2013; 13(1): 81. PMid:23537172 https://doi .org/10.1186/14 71-2393-13-81

[69] Ronsmans C, Etard JF, Walraven G et al. Maternal mortality and access to obstetric services in West Africa. Tropical Medicine \& International Health. 2003; 8(10): 940-948. PMid:14516306 https://doi.org/10.1046/j.1365-3156.2003.01111.x

[70] Kambala C, Morse T, Masangwi $S$ et al. Barriers to maternal health service use in Chikhwawa, Southern Malawi. Malawi Medical Journal. 2011; 23(1). https://doi.org/10.4314/mmj .v23i1. 6767 3

Published by Sciedu Press 
[71] Holloway I, Kathleen TG. In Gerrish K, Lathlean J. The research process in nursing. 7th ed. UK: John Wiley \& Sons, Ltd. 2015.

[72] Kyomuhendo GB. Low use of rural maternity services in Uganda: impact of women's status, traditional beliefs and limited resources. Reproductive Health Matters. 2003; 11(21): 16-26.

[73] Shaikh BT, Hatcher J. Health seeking behaviour and health service utilization in Pakistan: challenging the policy makers. Journal of public health. 2004; 27(1): 49-54. PMid:15590705 https: //doi.org/10.1093/pubmed/fdh207

[74] Matsuoka S, Aiga H, Rasmey LC et al. Perceived barriers to utilization of maternal health services in rural Cambodia. Health Policy. 2010; 95(2-3): 255-263. PMid:20060193 https ://doi.org/10.1 016/j.healthpol.2009.12.011

[75] Makama GA. Patriarchy and gender inequality in Nigeria: The way forward. European Scientific Journal, ESJ. 2013; 9(17).

[76] Lesejane D. Fatherhood from an African cultural perspective. Baba: men and fatherhood in South Africa. 2006; 173-182.

[77] Biratu BT, Lindstrom DP. The influence of husbands' approval on women's use of prenatal care: results from Yirgalem and Jimma towns, south west Ethiopia. Ethiopian Journal of Health Development. 2006; 20(2): 84-92. https://doi.org/10.4314/ejhd.v $20 i 2.10017$

[78] Birmeta K, Dibaba Y, Woldeyohannes D. Determinants of maternal health care utilization in Holeta town, central Ethiopia. BMC Health Services Research. 2013; 13(1): 256. PMid:23822155 https : //doi.org/10.1186/1472-6963-13-256

[79] Story WT, Burgard SA, Lori JR, et al. Husbands' involvement in delivery care utilization in rural Bangladesh: A qualitative study. BMC Pregnancy and Childbirth. 2012; 12(1): 28. PMid:22494576 https://doi .org/10.1186/1471-2393-12-28

[80] Maseno L, Kilonzo SM. Engendering development: Demystifying patriarchy and its effects on women in rural Kenya. International Journal of Sociology and Anthropology. 2011; 3(2): 45-55.

[81] Jokhio AH, Winter HR, Cheng KK. An intervention involving traditional birth attendants and perinatal and maternal mortality in Pakistan. New England Journal of Medicine. 2005; 352(20): 2091-2099. PMid:15901862 https://doi .org/10.1056/NEJMsa042830

[82] Downe S, Finlayson K, Walsh D et al. 'Weighing up and balancing out': a meta-synthesis of barriers to antenatal care for marginalised women in high-income countries. BJOG: An International Journal of Obstetrics \& Gynaecology. 2009; 116(4): 518-529. PMid:19250363 https://doi.org/10.1111/j.1471-0528.2008.02067.x

[83] Kupek E, Petrou S, Vause S, et al. Clinical, provider and sociodemographic predictors of late initiation of antenatal care in England and Wales. BJOG: An International Journal of Obstetrics \& Gynaecology. 2002; 109(3): 265-273. PMid:11950181 https: //doi.org/10.1111/j.1471-0528.2002.00524.x

[84] Afshar H. Women and empowerment: Illustrations from the Third World. 2016; Springer.

[85] Thulasi PT. Empowerment of Women. Commonwealth Publishers Pvt. Limited. 2014.
[86] Sharps PW, Campbell J, Baty ML, et al. Current evidence on perinatal home visiting and intimate partner violence. Journal of Obstetric, Gynecologic, \& Neonatal Nursing. 2008; 37(4): 480-491. PMid:18754987 https://doi.org/10.1111/j.1552-6909. 20 08.00267.x

[87] Barlow J, Stewart-Brown S, Callaghan H, et al. Working in partnership: the development of a home visiting service for vulnerable families. Child Abuse Review: Journal of the British Association for the Study and Prevention of Child Abuse and Neglect. 2003; 12(3): 172-189. https://doi.org/10.1002/car.796

[88] Vieira C, Portela A, Miller T, et al. Increasing the use of skilled health personnel where traditional birth attendants were providers of childbirth care: a systematic review. PLoS One. 2012; 7(10): e47946. PMid:23110138 https://doi.org/10.1371/journal.pone.0 047946

[89] Agus Y, Horiuchi S, Porter SE. Rural Indonesia women's traditional beliefs about antenatal care. BMC Research Notes. 2012; 5(1): 589. PMid:23106915 https://doi .org/10.1186/1756-0 500-5-589

[90] Davey MA, Brown S, Bruinsma F. What is it about antenatal continuity of caregiver that matters to women? Birth. 2005; 32(4): 262-271. PMid:16336367 https://doi.org/10.1111/j.0730-7659.20 05.00384.x

[91] Page L. One-to-one midwifery: restoring the "with woman" relationship in midwifery. The Journal of Midwifery \& Women's Health. 2003; 48(2): 119-125. https://doi.org/10.1016/S1526-952 3(02) 00425-7

[92] Biró MA, Waldenström U, Brown S, et al. Satisfaction with team midwifery care for low- and high-risk women: A randomized controlled trial. Birth. 2003; 30(1): 1-10. PMid:12581034 https: //doi.org/10.1046/j.1523-536X.2003.00211.x

[93] Jepsen I, Mark E, Nøhr EA et al. A qualitative study of how caseload midwifery is constituted and experienced by Danish midwives. Midwifery. 2016; 36: 61-69. PMid:27106945 https://doi .org/10.1 016/j.midw . 2016.03.002

[94] Hibbard JH, Greene J. What the evidence shows about patient activation: better health outcomes and care experiences; fewer data on costs. Health Affairs. 2013; 32(2): 207-214. PMid:23381511 https://doi.org/10.1377/hlthaff .2012.1061

[95] Forster DA, McLachlan HL, Rayner J, et al. The early postnatal period: exploring women's views, expectations and experiences of care using focus groups in Victoria, Australia. BMC Pregnancy and Childbirth. 2008; 8(1): 27. PMid:18644157 https ://doi .org/10 .1186/1471-2393-8-27

[96] Teate A, Leap N, Rising SS, et al. Women's experiences of group antenatal care in Australia - the Centering Pregnancy Pilot Study. Midwifery. 2011; 27(2): 138-145. PMid:19386402 https ://doi . org $/ 10.1016 / j$.midw . 2009.03.001

[97] Lewis G. Saving Mothers' Lives: the continuing benefits for maternal health from the United Kingdom (UK) Confidential Enquires into Maternal Deaths. In Seminars in Perinatology. 2012; 36(1): 19-26. PMid:22280861 https://doi.org/10.1053/j.semperi. 2011 .09 .005 